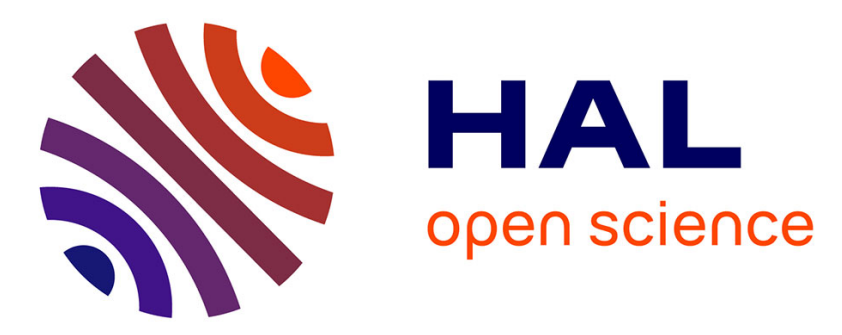

\title{
Exprimer son expérience scolaire dans la migration : une analyse des méthodes participatives
}

\author{
Maïtena Armagnague-Roucher, Isabelle Rigoni
}

\section{To cite this version:}

Maïtena Armagnague-Roucher, Isabelle Rigoni. Exprimer son expérience scolaire dans la migration : une analyse des méthodes participatives. La nouvelle revue - Éducation et société inclusives, 2018, 82, pp.27-45. hal-01799593

\section{HAL Id: hal-01799593 \\ https://hal.science/hal-01799593}

Submitted on 19 Nov 2018

HAL is a multi-disciplinary open access archive for the deposit and dissemination of scientific research documents, whether they are published or not. The documents may come from teaching and research institutions in France or abroad, or from public or private research centers.
L'archive ouverte pluridisciplinaire HAL, est destinée au dépôt et à la diffusion de documents scientifiques de niveau recherche, publiés ou non, émanant des établissements d'enseignement et de recherche français ou étrangers, des laboratoires publics ou privés. 


\title{
Exprimer
}

son expérience scolaire

dans la migration :

une analyse

des méthodes participatives

\author{
Maïtena ARMAGNAGUE \\ Maîtresse de conférences en sociologie \\ INSHEA, Grhapes (EA 7287) \\ Université Paris Lumières \\ Isabelle RIGONI \\ Maîtresse de conférences en sociologie \\ INSHEA, Grhapes (EA 7287) \\ Université Paris Lumières
}

Résumé: À partir de recherches fondées sur des projets relatifs à la scolarisation des enfants et jeunes migrants dans des établissements scolaires des premier et second degrés en Île-de-France et en province, l'article s'intéresse à la compréhension des expériences enfantines à l'école. Ces expériences sont conçues à la fois comme des données sociologiques et en même temps, comme productrices de théorisation. Ce parti pris nécessite une réflexion épistémologique et méthodologique ayant fait l'objet d'une mise en forme par l'intermédiaire de méthodologies visuelles et artistiques visant à restituer l'agency des jeunes enquêtés. Ces méthodologies mettent en exergue à la fois la centralité de la figure enseignante engageant l'autonomie des enfants et jeunes dans le système éducatif ainsi que le rapport normatif que ces enfants entretiennent à leur environnement scolaire, notamment vis-à-vis de leurs pairs. En outre, on apprend que des enfants restituent un cadre de perception segmenté de leur univers socio-scolaire.

Mots-clés: Expériences enfantines - Figure enseignante - Méthodes participatives - Migration - Normativité Scolarisation.

\section{Expressing school experience in migration: an analysis of participatory methods}

Summary: Based on research programs related to the schooling of children and young migrants in primary and secondary schools in France, the paper focuses on the understanding of children's experiences at school. These experiences are conceived both as sociological data and at the same time as producers of theorization. This bias requires an epistemological and methodological reflection that has been shaped by means of visual and artistic methodologies aimed at restoring the agency of the young people surveyed. These methodologies highlight both the centrality of the teacher figure engaging the autonomy of children and young people in the education system as well as the normative relationship that these children have with their school environment, particularly with respect to their peers. In addition, we learn that children restore a framework of segmented perception of their socio-academic environment.

Keywords: Children's experiences - Migration - Normativity - Participatory methods - Schooling - Teaching figure. 
L'École française scolarise des élèves migrants depuis plus d'un siècle, tout comme dans les autres pays où l'immigration est une réalité ancienne, corrélée au développement industriel. Lorsque la sociologie a commencé à s'intéresser aux familles en situation de migration et à leurs enfants, la place des élèves au sein de l'institution scolaire a principalement été pensée en termes distributifs. Plusieurs études d'Abdelmalek Sayad menées dans les années 1970 se sont attachées à démontrer l'inadéquation des structures éducatives aux conditions des enfants d'immigrés, ceci aboutissant à produire de la relégation et donc à mettre en échec la fonction distributive de l'École, les inégalités scolaires étant le reflet des inégalités sociales (Sayad, 2014). Plus tard, Claire Schiff (2003) s'est intéressée aux processus de déscolarisation des migrants. Du côté des sciences de l'éducation et de la didactique, ce sont principalement les fonctions éducatives qui sont au cœur de l'analyse (Mendonça Dias, 2012, 2013). De même, les linguistes se sont engagés dans de nombreux échanges autour de l'utilisation des langues maternelles et des langues de scolarisation (Cummins, 2009; Auger, 2010). En revanche, peu d'études ont porté sur les fonctions de socialisation à l'œuvre au sein de l'École et leur réception par les principaux intéressés, à savoir les élèves, spécifiquement les élèves migrants nouvellement arrivés désignés comme allophones. Leur condition est pourtant particulière tant au titre de leur migration, qui a donné lieu à plusieurs types de ruptures parfois radicales et pas toujours explicitées par les adultes de leur entourage, qu'au titre de leur allophonie, qui les place dans des situations d'apprentissage et de socialisation fragiles. II est donc urgent de s'interroger sur l'expérience scolaire de ces élèves et, plus particulièrement, sur la nature de leurs rapports sociaux.

Notre approche se rapporte ainsi à la sociologie compréhensive qui part de la subjectivité des acteurs. Dans la tradition de la sociologie de l'expérience (Dubet, 1994), nous cherchons à stimuler la perception que les acteurs ont de leurs propres (inter)actions. D'où la nécessité de penser des méthodes qui permettent au chercheur d'appréhender la conscience que les acteurs ont d'eux-mêmes et des autres et leurs rapports entre eux. II nous est donc apparu indispensable d'élaborer et d'expérimenter une méthodologie qui puisse renseigner l'expérience individuelle, mais aussi sociale, tout en nous décentrant pour comprendre les points de vue enfantins et juvéniles. Ainsi, notre article vise d'une part, à asseoir l'intérêt théorique de la méthodologie participative et, d'autre part, à la mettre en pratique en analysant les données recueillies.

\section{SORTIR D'UNE APPROCHE ADULTOCENTRÉE : UNE DÉMARCHE OUI S'INSCRIT DANS UNE POSTURE ÉPISTÉMOLOGIQUUE ET MÉTHODOLOGIOQUE PLUS LARGE}

Nos recherches sur l'insertion socio-scolaire des enfants et des jeunes migrants s'inscrivent dans le cadre de plusieurs programmes menés dans des établissements scolaires de premier et second degrés, auprès d'élèves scolarisés en classe ordinaire et/ou dans des Unités pédagogiques pour élèves allophones nouvellement 
arrivés (UPE2A ${ }^{1}$ ). Nos enquêtes ont porté sur 8 UPE2A et 14 classes ordinaires dans 10 établissements dans lesquels nous étions présentes une demi-journée hebdomadaire pendant 5 à 12 semaines selon les établissements en dispositif UPE2A ou en classe ordinaire.

Prendre en compte le point de vue des enfants et des jeunes migrants pour comprendre leurs expériences socio-scolaires nécessite de les considérer comme des sujets certes enfantins, mais agissant et non pas comme de simples objets d'enquête, ce qui reconfigure profondément la relation d'enquête (Sirota, 2012). La captation de moments permettant de saisir les ressorts de la participation socio-scolaire de ces élèves implique la conception de méthodes adaptées dont la réalisation rend indispensable la collaboration entre l'enseignant, le chercheur et éventuellement I'intervenant extérieur participant à l'investigation (Armagnague, Cossée et al., 2017). Dans l'application de ces méthodes adaptées et dans l'analyse de leurs résultats, il convient également de prendre en compte les difficultés de l'expression enfantine et juvénile, qui pourront être contournées par d'indispensables séances d'observation.

\section{Enfants et jeunes : objets et surtout sujets d'enquête}

L'enquête auprès de jeunes et d'enfants migrants désignés allophones, dont les origines sont plurielles et diverses, pose dès le départ la question des modalités communicationnelles au chercheur qui ne peut jamais maîtriser toutes les langues parlées par les enfants auprès desquels il enquête. Outre la question de la langue, le chercheur représente un acteur éloigné de l'univers enfantin car c'est un adulte, de catégorie socioprofessionnelle supérieure. Dans un contexte où l'altérité du chercheur s'impose de fait, il importe de tenir pour acquis cette extranéité et d'en faire le point de départ d'une posture méthodologique pensée pour construire une relation réciproque avec les enquêtés. Ces modalités communicationnelles sont d'autant plus en enjeu crucial qu'elles interrogent des dimensions éthiques et déontologiques. Dans bien des domaines, travailler sur les problématiques scolaires se fait souvent sans les principaux concernés: les enfants et les jeunes. On teste alors sur eux l'efficience de nouveaux outils, on mesure à partir de leurs pratiques et productions des réalités correspondant à des hypothèses que le ou les chercheurs auront bâti dans des espaces de connaissance académique. Des chercheurs tenteront de convaincre que le haut niveau de technicité qu'ils promeuvent parce qu'ils arrivent à le maîtriser est nécessairement le garant d'une mesure toujours plus fine des objets observés, donc de la restitution de la réalité. Mais au fond, une telle posture n'a pas encore prouvé qu'elle dévoilait plus précisément les mécanismes socio-scolaires et son affirmation à l'objectivité ne permet, par ailleurs, pas systématiquement de penser des formes éducatives plus justes et plus efficaces. Mais elle a assurément démontré son haut niveau d'expertise et d'inaccessibilité quand elle n'est pas comprise par les intéressés sur lesquels porte l'enquête. Ainsi, certaines recherches sont déployées à partir de méthodes n'ayant pas été explicitées aux enquêtés alors qu'elles sollicitent

\footnotetext{
1. Les programmes de recherche sont Migriti (2014-17, financé par I'Université Paris Lumières), Evascol (2015-18, financé par le Défenseur des droits), Alterecole (2016-19, financé par la région Aquitaine) et Educinclu (2016-19, financé par l'Ires).
} 
directement leur participation voire qu'elles prétendent travailler de façon qualitative sur les dimensions expérientielles de leur scolarité. Pourquoi certaines enquêtes fonctionnent-elles ainsi? Probablement parce que l'enquêté peut avoir, d'un point de vue éthique, méthodologique et épistémologique, trois statuts: celui de donnée à observer, restituer et analyser; celui de producteur de données, ce qui impose au chercheur d'analyser avec réflexivité les conditions de production de ces données par l'enquêté; enfin, le statut de producteur de théorie, ce qui demande au chercheur de partir de ce qui est livré et l'empêche de sélectionner ou de tordre son matériau d'enquête. La plupart des recherches qualitatives en sciences humaines et sociales sur l'école tiennent compte des conditions de production des récits (Kaufmann, 2016) des jeunes enquêtés, mais très rares sont celles qui considèrent ces récits comme le point de départ épistémologique et méthodologique de la production du savoir les concernant (Touraine, 1978). Dans nos recherches, nous avons fait le choix de donner aux enquêtés, fussent-ils jeunes et parfois non-francophones, le statut de coproducteurs des conceptualisations que nous formulions, ce qui nous a engagé à utiliser des méthodologies collaboratives (Armagnague et Rigoni, 2017) notamment ancrées dans la sociologie visuelle (Becker, 1974) et parfois à en concevoir de nouvelles, à partir d'hybridations expérimentales, co-gérées et animées par des chercheurs et des artistes (Armagnague, Cossée et al., 2017).

\section{L'importance de la méthode adaptée}

La volonté de saisir au plus près l'expression enfantine ou juvénile en tant que telle, indépendamment de la désignation de l'intéressé en simple élève, nous demandait de concevoir l'enfant ou le jeune en tant que sujet d'analyse sociologique et ceci y compris dans le cadre scolaire où l'on se focalise souvent sur la figure de l'élève (Sirota, 2005). L'enjeu dans ce contexte est à la fois de susciter l'expression des enquêtés, tandis que spontanément, les jeunes et, plus encore les enfants, ne se livrent pas à de longs récits, sans pour autant verser dans des formes factices ou standardisées de production dans lesquelles l'enquêté produit en anticipant un attendu inféré (un beau dessin, une histoire ayant une belle morale, un texte suscitant l'émotion ou un texte valorisable scolairement). Notre objectif étant d'adopter le point de vue de l'enfant, de lutter contre les tentations d'adultocentrisme (Delalande, 2007; Tersigni, 2014) et en tenant compte du contexte organisant le recueil des données (Becker, 2001), nous avons cherché à concevoir des méthodes d'enquête permettant aux enquêtés de s'exprimer en lien avec nos thématiques de recherche tout en faisant comprendre aux enfants et adultes que nous ne visions, par ces méthodes, ni l'utilité scolaire ni le réconfort psychologique, ni l'esthétisme, cher aux enseignants et aux artistes. Par l'intermédiaire d'ateliers de clown-théâtre, de théâtre-forum, de créations plastiques, de dessins et de photographies, ainsi que de séances d'écriture, les enfants et jeunes s'emparaient d'une consigne de façon individuelle et dans un temps court afin que les réalisations ne soient ni comparées ni retouchées. Les enseignants, présents dans ces ateliers ne jugeaient ni n'évaluaient ces productions. Ils ne les conseillaient ou ne les orientaient pas non plus. Les consignes données étaient le fruit d'un travail de déconstruction et de traduction de notre problématique conduit par les sociologues et les artistes, dont l'une était 


\section{DOSSIER}

aussi chercheure en formation². La mise en forme des saynètes, des photographies ou encore des dessins offraient d'appréhender les choix et stratégies déployés par les enfants pour nous livrer leur quotidien lié à l'école, les termes utilisés, les objets représentés, les mots utilisés et le répertoire sociologique référé permettaient de saisir les illustrations socialisantes et socialisatrices. Enfin, chaque réalisation s'accompagnait d'une restitution partagée: explicitation de sa production, réponse aux questions formulées par les pairs élèves, sélection des questions et arbitrages dans les modalités de réponse. Dans ces moments, il était question d'échanger avec les pairs élèves. L'artiste et le sociologue ayant des fonctions de reformulation, d'approfondissement ou de clarification des énoncés. Ces échanges avaient pour effet de formaliser des réflexivités enfantines et juvéniles circonscrites, mais défendues par les intéressés. Ainsi, les points de vue étaient débattus avec les camarades, en classe entière ou par demi-groupes, ce qui pouvait donner lieu à des oppositions entre enfants et jeunes, à des reformulations et à des dialogues que le cadre formel de la classe ne permet pas.

\section{L'importance du sociologue et de l'enquête}

Le chercheur qui enquête dans des conditions ethnographiques auprès d'enfants et de jeunes en instituant de telles approches n'est pas sans savoir qu'il élabore de toute pièce des modalités de participation et d'expression qui n'existent pas sans cette construction. Ceci exige qu'il s'inscrive dans une posture propre à l'analyse critique et réflexive de ce que constitue l'artefact créé par la recherche et par l'implication du chercheur et la dimension potentiellement superficielle et/ou factice de cette démarche. Car dans la quotidienneté scolaire des enfants migrants, les questions sociales font rarement l'objet d'un temps dédié par l'institution scolaire. En conséquence, cet espace est alternativement et quelquefois tout à tour surinvesti par quelques enfants et jeunes ou au contraire il suscite des réserves de la part d'autres enquêtés qui y participent de façon plus timorée, exprimant à ce titre une gêne voire, dans quelques cas, une opposition. Ces figures hétérogènes d'investissement révèlent parfois des besoins de reconnaissance de la part d'enfants ou de jeunes en situation de minorisation. Elles traduisent également des formes différentes d'expression sociale et, au-delà, de rapport même à l'expression rendue publique, hétérogénéité constitutive et illustrative des situations sociologiques elles aussi composites dans les terres d'émigration et dans l'immigration: les enfants et jeunes migrants ne viennent pas des mêmes milieux sociaux, n'ont pas les mêmes contextes d'arrivée et de vie en France. Dans certains cas, leur parole dans le projet familial a peu de place (Rigoni, 2017). II est notable que dans chacune de ces expressions, c'est bien la présence de l'enquêteur qui configure un système relationnel et interactionnel inédit. Le sociologue, parce qu'il cherche dans ce contexte à favoriser les conditions de formulation du matériau révélateur d'une inscription des individus dans la stratification sociale, dans des positions conditionnant leurs relations sociales et leur rapport à l'institution scolaire, détient un rôle déterminant. On aurait tort de croire

2. II s'agit de Nancy Lallouette, co-auteure d'un article académique exposant cette méthodologie expérimentale (Armagnague, Cossée et al., 2017). 
que cette centralité du chercheur n'est perceptible que par lui seul. Les enquêtés accordent au sociologue une place centrale dans l'échange y compris si celui-ci $n$ 'intervient pas directement. Les enfants et jeunes interagissent entre eux, mais regardent le chercheur, tentent parfois de capter son attention voire le sollicitent explicitement. Nombre de ces enquêtés posent des questions lorsque le chercheur se présente pour la première fois en classe, demandent ce à quoi " serviront " les notes prises par les enquêteurs et aiment à regarder ce qui est noté. D'ailleurs, si l'évocation de l'enquête ne fait l'objet d'aucune saynète conçue par les enfants et jeunes, le sociologue est systématiquement dessiné lorsqu'il est demandé aux jeunes d'illustrer leur quotidien scolaire. Ainsi, alors qu'il ne venait en classe qu'une fois par semaine ${ }^{3}$, la sociologue est-elle dessinée dans la classe ou à côté d'elle, dans la cour de récréation en position d'importance équivalente à l'enseignant ou à côté de lui alors que sa présence dans l'établissement et auprès des enfants et jeunes est moindre. De même, le sociologue qui utilise la photographie comme outil méthodologique est-il lui-même pris en photo par certains jeunes, dans une appropriation symétrique des outils visuels d'enquête.

Cette centralité du sociologue dans les productions des enquêtés témoigne du lien qu'ils font entre l'expression sur des sujets à la fois relativement communs et presque jamais sondés et l'opportunité qui est donnée de le faire associée à la figure du chercheur.

3. Précisons que cette présence d'une demi-journée par semaine dans chaque établissement durant l'ensemble d'une année scolaire (10 mois) constitue une mise en relation importante pour les enfants et les jeunes, qui de fait nous connaissent, viennent à notre rencontre dans l'école ou le collège, mais aussi à l'extérieur, dans le centre-ville par exemple. 


\section{DOSSIER}

Illustration 1 : Dessin d'une fille de 8 ans, en UPE2A, région nord de Paris répondant à la consigne " Dessine des lieux de l'école ".

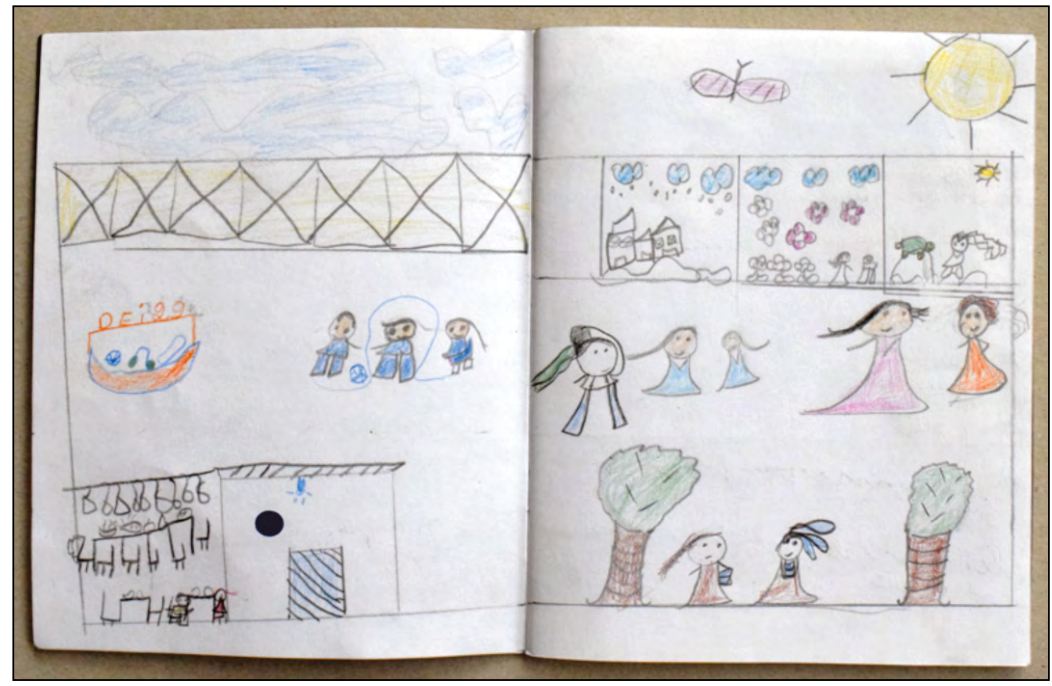

Légende de l'illustration 1: La cour de récréation est représentée.

Y figurent, page de droite : la fillette et sa meilleure amie (les deux personnages en bleu), deux maîtresses surveillant la récréation (en bas, personnages marrons entre les deux arbres),

la maîtresse d'UPE2A (à droite, personnage orange) discutant avec la sociologue (personnage à droite en rose).

Illustration 2: Dessin d'un garçon de 9 ans en UPE2A, région nord de Paris répondant à la consigne " Dessine des lieux de l'école ".

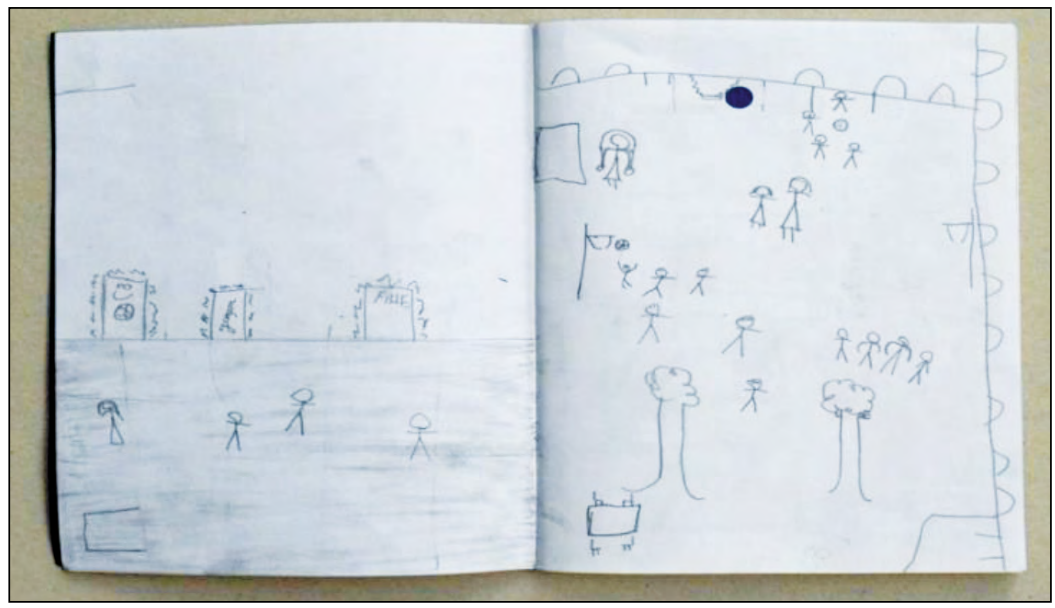

Légende de l'illustration 2: La cour de récréation est représentée.

$Y$ figurent, page de droite au milieu en haut deux femmes:

I'une est décrite comme étant l'enseignante, l'autre la sociologue. 


\section{Enjeux et difficultés de l'expression enfantine}

Dans ce contexte, une des difficultés du chercheur va être de mettre en perspective ce qui est révélé dans le cadre de méthodologies participatives avec la réalité sociale plus générale des enquêtés. En ce sens, les méthodes participatives ne peuvent que compléter des approches ethnographiques de long terme et impliquées (Sirota, 2012). Car il sera nécessaire de réaliser une sorte d'étalonnage de ce qui est exprimé, parfois avec force dans des ateliers d'expression théâtrale, écrite ou d'arts plastiques et ce qui relève de la vie socio-scolaire ordinaire, dans laquelle la place de l'expression libre est marginale. Ainsi, I'usage de matériaux choc (induisant du pathos ou de la colère) est un véritable risque pour le sociologue; il ne pourra le manier qu'avec prudence et à l'aune d'une relation d'enquête parfaitement maîtrisée car il ne peut faire l'économie de garder en tête combien de tels matériau sont profondément situés, produits dans et parfois par un contexte d'enquête spécifique (Becker, 2001). Nous avons alors fait le choix de mettre chaque production en perspective de nos observations régulières en classe, ainsi que dans certains cas d'entretiens, et de n'accorder aucune signification psycho-symbolique ou morale autre que celle qui nous était livrée par l'auteur de la saynète ou de la production artistique lui-même. Partant de là, que nous enseignent les réalisations enfantines et juvéniles, entendues comme des points de vue situés sur leur propre quotidienneté?

\section{RESTITUTIONS D'EXPÉRIENCES SOCIO-SCOLAIRES}

L'élaboration et l'expérimentation de ces méthodes adaptées nous a permis de recueillir un riche matériau qui nous a ouvert la voie vers une compréhension des multiples expériences socio-scolaires (Dubet, Martuccelli, 1996) des enfants et des jeunes migrants. Ces outils méthodologiques construits ont permis d'entrer en interaction et en communication avec les élèves et de les accompagner vers une production libre d'expression orale, corporelle ou encore écrite. Ainsi, le choix des thèmes d'expression a-t-il été largement réalisé par les enfants et les jeunes eux-mêmes, les sociologues privilégiant leur émergence spontanée, comme dans l'esprit du théâtre-forum et du clown-forum. Les principaux thèmes qui ont émergé des expériences socio-scolaires des élèves migrants concernent le rapport aux enseignants, la socialisation avec leurs pairs et le parcours scolaire.

\section{La figure de l'enseignant en classe ordinaire}

Chaque élève allophone nouvellement arrivé bénéficiant d'un soutien en UPE2A est inscrit en classe ordinaire, qui constitue son rattachement officiel, et doit y suivre une partie des enseignements. Depuis la fin des années 1990, dans l'esprit de la promotion de l'intégration et de la diversité à l'école, l'institution scolaire tend à favoriser l'instauration de dispositifs " ouverts" et ayant lieu pendant le temps scolaire (Armagnague, Rigoni, 2016). En ce sens, les circulaires de $2012^{4}$ relatives aux élèves allophones et aux élèves itinérants et du voyage réforment les CLIN et

4. Circulaires n² 2012-141, 2012-142 et 2012-143 parues au Bulletin officie/ n 37 du 11 octobre 2012. 


\section{DOSSIER}

les CLA, jugées trop ségrégatives car fermées, au profit de dispositifs plus ouverts (les UPE2A ${ }^{5}$ et les UPS) dans lesquels les élèves à besoins particuliers sont pris en charge au minimum 9 heures par semaine dans le premier degré et 12 heures hebdomadaires dans le second degré. En dehors des heures passées dans le dispositif, les élèves doivent intégrer les enseignements de leur classe ordinaire. Les applications diffèrent fortement selon les établissements, les circonscriptions et les académies et l'on observe sur le terrain des réalités d'inclusion très diverses. Dans certains cas, plus souvent dans le second degré, les élèves sont inclus a minima dans leur classe ordinaire de rattachement. Dans d'autres cas au contraire, particulièrement dans le premier degré et lorsque les dispositifs UPE2A sont mobiles (ce qui conduit l'enseignant à intervenir dans plusieurs établissements), les élèves suivent davantage de cours en classe ordinaire.

Les situations d'inclusion constituent une expérience vécue de façon très hétérogène par les élèves migrants allophones. Dans ce contexte, la figure de l'enseignant en classe ordinaire est particulièrement prégnante, souvent abordée dans les productions et les propos des élèves, particulièrement dans le premier degré. Dans nos enquêtes, le théâtre-forum a permis d'aborder le rapport avec l'enseignant en classe ordinaire. Prenons l'exemple d'une école où les séances ont été menées auprès des élèves d'une classe de CM2 incluant ponctuellement plusieurs élèves allophones. Les séances ont eu lieu pendant le temps scolaire, mais en dehors de la présence de l'enseignante, dans la salle polyvalente de l'école. Une compagnie spécialisée dans le théâtre-forum est intervenue pendant dix séances, dans le cadre d'un projet porté par une association partenaire de I'un de nos programmes de recherche, avec la collaboration de la sociologue. Lors des séances de préparation, les professionnels du théâtre-forum et la sociologue ont tenté de cerner les questions et les situations problématiques que les élèves souhaitaient mettre en avant. Les situations de classe ont constitué I'un des trois thèmes avancés par les élèves, qu'ils soient migrants ou non. Lors des saynètes, la figure de l'enseignant est rapidement apparue comme un élément central du quotidien des relations en classe, plus que le rapport avec les pairs. Les premières saynètes portaient pourtant sur la relation d'entraide entre élèves: un élève ordinaire est placé à côté d'un élève migrant allophone, tous deux faisant face à l'enseignant qui leur demande un travail (image 1). II s'agissait d'observer si une situation d'entraide allait se mettre en place entre les deux élèves, et de quelle nature. Cependant, au fur et à mesure des saynètes, la relation entre les deux élèves a été rapidement occultée au profit des rapports entretenus avec l'enseignant qui, finalement, est apparu comme le personnage central cristallisant l'ensemble des rapports socio-scolaires dans I'espace classe. Le rôle de l'enseignant a dès lors été joué par plusieurs élèves volontaires à tour de rôle, plutôt que par l'intervenante de théâtre-forum. Face à l'élève incarnant l'enseignant, ce ne sont plus des enfants qui jouent le rôle

5. Seules les UPE2A-NSA, destinées aux élèves non scolarisés antérieurement, fonctionnent en circuit fermé, ceux-ci n'étant pas inscrits en classe ordinaire, bien que pouvant y suivre quelques enseignements afin de compléter I'horaire scolaire, notamment en EPS, arts plastiques ou musique. 
des élèves, mais les intervenants de théâtre-forum (image 2). En délaissant le rôle des élèves pour s'emparer de celui de l'enseignant, les élèves enquêtés signifient l'importance capitale du rôle et de la fonction de l'enseignant à leurs yeux. Leur mise en scène et leurs expressions indiquent également qu'ils ont des éléments à apporter sur la manière d'agir des enseignants, sur la façon dont ceux-ci les considèrent et interagissent avec eux.

\section{Image 1: Saynète de théâtre-forum dans une classe de CM2 d'une école du Sud-Ouest de la France}

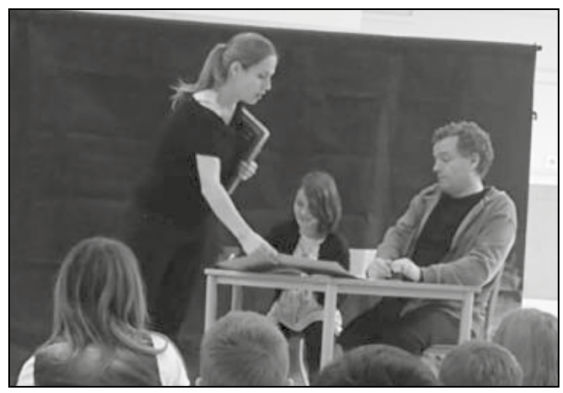

Légende de l'image 1 : cette saynète représente un moment de classe.

Les deux personnes attablées incarnent un binôme élève migrant allophone / élève non-migrant.

Image 2 : Saynète de théâtre-forum dans une classe de CM2 d'une école du Sud-Ouest de la France

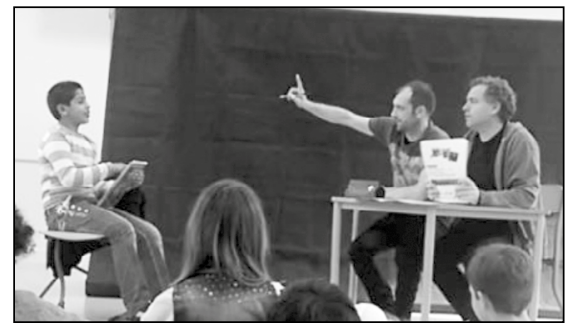

Légende de l'image 2 : cette saynète reptésente un moment de classe.

Les deux acteurs attablés incarnent un binôme élève migrant allophone / élève non-migrant, tandis que l'enfant assis face à eux s'approprie le rôle de l'enseignant.

Dans un atelier de clown-forum réunissant des élèves migrants allophones du premier degré pendant le temps périscolaire d'un autre établissement, la consigne était « un enfant d'un autre pays arrive dans la classe, que se passe-t-il? ». Cette question était le fruit d'une réflexion collaborative entre la sociologue, l'artiste clown et les enfants qui décrivaient l'enseignant comme une figure centrale dans l'école, mais avec lequel les échanges étaient parfois difficiles, arrivant dans certains cas à des situations de blocage. Le clown-forum constituait donc ici un outil particulièrement adapté à la 


\section{DOSSIER}

fois pour faire émerger cette question et pour tenter d'y apporter collectivement des éléments de réponse. Lors des cinq saynètes jouées par les élèves, les garçons ont toujours attribué au maître un rôle directif et parfois peu ou pas accueillant. Dans l'une des saynètes, le nouvel élève entre dans la salle de classe, apeuré; le maître ne l'accueille pas puis lui parle par signes et finit par le mettre dans un coin en disant: " ah, parfait, tu seras tranquille! ». Lorsqu'une fille du groupe d'enfants a voulu incarner le rôle d'une maîtresse bienveillante, cela a conduit les garçons à se plaindre de favoritisme envers le nouveau venu:

Yvan', jouant le rôle d'un enfant joyeux, exprime pourtant à cette occasion son mécontentement: " je vais me plaindre au directeur, elle favorise toujours les nouveaux!"

Titouan, jouant le rôle d'un élève sérieux, ajoute alors: " j'ai envie de rentrer chez moi! »

Dans cet atelier, aucun enfant n'a véritablement choisi de jouer le rôle d'un enseignant en posture d'accueil, aidant, expliquant, accompagnant le nouvel élève migrant. Nous pourrions évoquer en contrepoint des expériences bien différentes d'élèves avec leur enseignant de classe ordinaire, tant celles-ci sont hétérogènes. Ce qui nous importe plutôt ici est de mesurer la centralité de l'enseignant dans les expériences des élèves au sein de l'espace classe, tandis que les relations entre élèves, pourtant souvent placés côte à côte ou en petits groupes formant des îlots et donc amenés à travailler ensemble, apparaissent secondaires.

\section{L'adulte référent: l'enseignant d'UPE2A}

Si dans leur ensemble, les enfants en âge d'être scolarisés en élémentaire accordent traditionnellement une place essentielle à leur enseignant, au respect de l'ordre scolaire que ce dernier impulse, ceci n'est traditionnellement plus le cas au collège où la communauté de semblables prend une part plus importante dans l'expérience scolaire (Dubet, Martuccelli, 1996) et où une large part de la socialisation juvénile se fait en dehors de l'école (Barrère, 2011). Mais dans le cas des enfants et des jeunes scolarisés en UPE2A, la centralité de la figure d'autorité éducative se prolonge jusqu'au collège. Lorsque les jeunes collégiens concevaient des saynètes relatives à leurs journées ou semaines scolaires, plus de la moitié d'entre eux référaient directement, dans la mise en scène, à une interaction avec I'enseignant d'UPE2A et, plus rarement avec le conseiller d'orientation. Ces adultes, représentés comme des guichetiers expliquant des fonctionnements scolaires à d'autres élèves ou comme faisant la morale, parfois criant et punissant, illustrent des relations fréquentes et proches avec l'adulte référent, mais marquées par un lien de dépendance. D'ailleurs, dans ce cadre, le clown était un outil offrant la liberté de grossir les caractéristiques burlesques de certains inter-actants: les élèves qui ne comprennent rien des énoncés d'examens ou des explications du conseiller d'orientation, des enseignants se mettant à vociférer particulièrement fort sur des

6. Les prénoms ont été modifiés pour préserver l'anonymat. 
élèves, ce qui suscite le rire du public. Ainsi voit-on la mise à distance réflexive quant à sa propre restitution de son quotidien social.

Chez les écoliers migrants désignés allophones, la figure de l'enseignant d'UPE2A apparaît sur quasiment tous les dessins, représenté au milieu ou à proximité des enfants. Cette figure personnalise les lieux et objets de l'école et les humanise. Dans le premier degré, tout se passe comme s'il s'opérait une incarnation des enjeux scolaires dans la figure de l'enseignant d'UP2EA: il est représenté comme faisant partie des différents lieux et, lors des restitutions faites par les enfants, il apparaît que la présence de l'enseignant dans une situation est ce qui, aux yeux des enfants, lui confère une qualité scolaire. Au-delà de cette dimension labelisante, I'enseignant d'UPE2A assure une fonction de sécurisation du parcours scolaire en lui donnant un caractère identifiable et donc anticipable. Cet enseignant est souvent identifié par les écoliers comme une personne empathique dont le rôle est d'aider à la réussite linguistique et plus largement scolaire, mais aussi, dans un autre registre, au développement individuel et à la relation de confiance. Cette vision d'une centralité bienveillante de l'enseignant d'UPE2A confère par ricochet à l'espace de la salle de classe un caractère de cocon que certains élèves ont parfois du mal à quitter. Que ce soit en école ou au collège, certains élèves demandent d'y rester plutôt que de descendre dans la cour de récréation voire même de ne pas rejoindre leur classe ordinaire pour continuer un travail qu'ils considèrent comme plus rassurant ou plus valorisant dans I'UPE2A. De nombreuses fois, alors que nous étions en observation en classe, nous avons constaté que des collégiens ayant été scolarisés en dispositif UPE2A l'année précédente, frappaient à la porte de I'UPE2A et demandaient à y rester quelques heures, expliquant ne pas être à l'aise dans une situation d'enseignement en " classe ordinaire".

À des niveaux différents du système éducatif, l'enseignant d'UPE2A apparaît donc comme central dans les expressions enfantines et juvéniles quant à leur expérience scolaire. Toutefois, la fonction de label rassurant au niveau élémentaire se dilue au collège où c'est aussi dans certains cas une relation de domination plus directement vécue et restituée qui se donne à voir. Cette situation est d'autant plus marquante que les liens de camaraderies scolaires apparaissent relativement ambivalents.

\section{Se socialiser aux pairs : répondre à une norme de camaraderie}

Dans les productions enfantines, la place des pairs est ambiguë. Soulignons d'abord que lorsqu'il n'a pas été demandé aux enquêtés de représenter leurs amis ou leurs camarades de classe, beaucoup l'ont quand même fait en dessinant les lieux de l'école les plus fréquentés ou leur classe. Les méthodologies participatives ont permis de faire émerger des informations que l'observation seule ne délivrait pas: des liens déclarés d'amitié avec des enfants de la classe de rattachement dans le premier degré (liens peu visibles dans la cour de récréation), mais aussi l'importance dans l'expérience scolaire de certaines amitiés pouvant a priori paraître plus ordinaires. Dans le premier degré, certains enfants ont dessiné la figure du ou de la meilleur-e ami-e fortement valorisée lors des restitutions collectives. Être dessiné donnait alors un statut public de reconnaissance et de loyauté, ce qui a suscité le mimétisme d'autres enfants. Loin d'être une seule expression factice due au mimétisme, 


\section{DOSSIER}

ceci témoigne de l'importance d'être considéré par celui que l'on estime être le plus proche de soi: on veut le satisfaire et ne pas le décevoir. Ainsi s'exprime le développement de formes de solidarités enfantines et juvéniles. Dans le même temps et dans ce contexte, nous avons constaté que certains enfants, pour ne pas se sentir isolés à produire différemment dans ce domaine, ont ajouté ex post des camarades sur leur dessin, afin de ne pas être les seuls à ne pas être en mesure d'expliciter un système de réciprocité élective à l'école. Dans leur cas, même si le fait de représenter des camarades n'a pas été spontané, il advient dans le huis-clos de la salle quand se prépare mentalement et socialement la restitution. Notons que ce type d'ajouts de seconde main ont exclusivement concerné la thématique de la camaraderie. Aucune autre n'a fait l'objet de tels réajustements.

À l'inverse, dans certains établissements, la question des relations entre pairs a été explicitement posée. Ainsi, dans une école de centre-ville comptabilisant de 20 à 40 \% d'élèves allophones selon les classes, les ateliers de théâtre-forum organisés avec les élèves d'une classe de CM2 portaient sur le thème de l'accueil dans trois espaces: la salle de classe dont nous avons vu que l'élément central était plutôt la figure de l'enseignant que celui des relations entre pairs; la cour de récréation; la cantine. Lors des séances préparatoires, des séquences de narration-débat ont mis en lumière des tensions issues de regroupements communautaires ou linguistiques dans les espaces communs de l'école:

Élève 1 (non-migrant): "Le plus grand souci dans la cour de récré, c'est quand il y a plusieurs petits groupes. Ils sont ensemble, veulent jouer ensemble mais après ils ne veulent pas et partent chacun de leur côté. "

Animatrice théâtre-forum: "Ils parlent en français?"

Élève 1: "Pas toujours. Alors qu'ils n'ont pas le droit! Les maîtres et maîtresses leur disent de parler en français. Après ils peuvent dire des choses pas bien. Mais c'est pas leur faute non plus, c'est automatique, ils n'arrivent pas à parler la langue française. "

Élève 2 (non-migrant): "Mais quand même si, ils peuvent essayer, sinon ça voudrait dire que tout est automatique, qu'ils sont des robots. Tout n'est pas automatique chez eux, ils ont une envie parce qu'il y a des trucs secrets à se raconter, ils ont envie qu'on ne les comprenne pas. "

Non seulement la part des élèves allophones est importante dans cette école, mais ceux-ci sont également en proportion importante de même origine linguistique, ce qui conduit parfois à des frictions entre élèves dans la cour de récréation, lorsqu'il leur est difficile d'entrer en communication et de partager des jeux. Toutefois, d'autres élèves non-migrants portent un regard empathique sur la condition des élèves en situation d'allophonie:

Animatrice théâtre-forum: "Est-ce que c'est dur de ne pas comprendre la langue française?"

Élève (non-migrant): "Oui. »

Animatrice théâtre-forum: "Pourquoi?" 
Élève (non-migrant): "Parce que ce n'est pas leur langue maternelle, qu'ils ont commencé à apprendre une autre langue [bulgare et/ou turc]. Mais aussi, je peux les comprendre les enfants qui ont envie de rester entre eux, parce qu'ils se sentent seuls autrement, et nous je pense qu'on ferait pareil si on arrivait dans un autre pays et qu'on trouvait des gens de notre pays à nous, on préfèrerait rester avec eux pour savoir à qui on parle, pour ne pas se sentir différent, et seul, et triste."

Mais le débat n'est pas tranché et la position exprimée par cet élève illustre des ambiguïtés difficiles à résoudre: "Moi, j'aimerais bien que tous les groupes se mélangent, parce que vraiment c'est inutile, tout le monde se comprend ici! Ça fait un bon p'tit bout de temps qu'ils sont ici, et ils veulent quand même rester de leur côté, ils veulent jamais se mélanger. Mais c'est pas que de leur faute, c'est aussi de la nôtre. Et donc j'aimerais bien que tout se mélange, que ce ne soient plus des groupes séparés, quoi. " Ce qui conduit l'animatrice de théâtre-forum à poser une question propice à la recherche d'hypothèses et de solutions : " comment faudrait faire pour que tout le monde se mélange? "Conformément aux principes du théâtre-forum tels que conceptualisés par Augusto Boal (1996), ce préalable séquentiel sur la narration-débat a permis de dégager des thèmes qui ont ensuite été retenus dans le cadre de la réalisation des saynètes, jouées par les élèves avec la participation de la compagnie de théâtre-forum.

Enfin, ailleurs encore, la sociologue a enquêté sur les relations amicales à la fois en classe ordinaire et au sein des dispositifs UPE2A. Un questionnaire a été proposé aux élèves de six classes de CM2 dans quatre établissements abritant également chacun une UPE2A. Les questions ont été élaborées après observation de moments de classe et discussion avec les élèves. Ouvertes, elles portaient sur le thème très général de l'amitié, afin de ne pas induire d'orientation relative aux situations d'altérité culturelle et/ou linguistique. Ce questionnaire a été accompagné dans chaque classe de séances de discussion en groupe (en classe entière ou en demi-groupes) menées par un binôme composé de deux chercheures ${ }^{7}$. Lors de ces séances, le principe était que le groupe lui-même crée les interactions, le binôme de chercheures régulant les échanges sans pour autant les maîtriser (Garcia, Hegel, 2011).

L'élément principal avancé par les élèves dans leurs productions écrites a été les relations filles-garçons, tandis que la socialisation entre enfants de différentes origines et la question de l'altérité culturelle et/ou linguistique n'ont été que marginalement évoquées. Lorsqu'elles l'ont été, c'était quasi-exclusivement par des élèves allophones pour exprimer leurs difficultés à entrer en communication avec les autres élèves, au moins dans les premiers mois de leur arrivée, lorsqu'ils ne maîtrisaient pas ou peu le français. Dans les séances en focus groupe en revanche, le binôme de chercheures a explicitement favorisé des moments d'échanges sur la socialisation entre élèves migrants allophones et élèves non-migrants. Partant toujours des productions écrites des élèves, dont certaines ont été citées anonymement pour

7. Alexandra Clavé-Mercier a fait partie de ces binômes dans 3 établissements de l'enquête. Dans la quatrième école, le binôme n'a pu être constitué et la sociologue est intervenue seule. 


\section{DOSSIER}

lancer le débat (par exemple, "Une amitié, ça se fait facilement, tu vas voir les autres. ", "La différence ne compte pas, on est tous pareils. ", " I faut découvrir tout le monde. " ou, au contraire, "Plus t'as d'amis, plus t'as de problèmes." , "On se dispute parce qu'on n'a pas la même opinion. "), les discussions en groupe ont ainsi conduit les élèves à s'interroger sur la possibilité d'être ami sans parler la même langue; à réfléchir aux raisons pour lesquelles parfois, dans la vie, les gens ne se parlent pas; pourquoi certains se moquent des autres; est-on plutôt amis avec des gens qui nous ressemblent...

Enfin, la socialisation entre pairs a lieu également en dehors du cadre scolaire. Des écoliers ont exprimé avoir autant d'amis à l'extérieur que dans leur école. La socialisation dans le quartier ainsi que dans l'espace familial ou communautaire joue un rôle tout aussi central pour certains enfants. Toutefois, avec les élèves de $\mathrm{CM} 2$, lorsque l'imminence de l'entrée au collège est évoquée, l'élément récurrent est la perspective de la séparation avec les camarades ou les amis qui rejoindront un autre collège du fait de la sectorisation. Cette période charnière dans la vie de tous les élèves prend un tour parfois déstabilisant pour certains enfants qui ont déjà connu des séparations affectives du fait de leur migration.

Les collégiens en UPE2A ont, quant à eux, bien davantage livré une vie sociale juvénile extra-scolaire dans les mises en scène proposées : évoquant les camarades du foyer, du quartier ou les amis liés à la famille ou au pays d'origine. Mis à part trois binômes de jeunes, lorsque le réseau électif est mentionné (sans incitation explicite à le faire), il réfère bien davantage à ce qui existe en dehors de l'école et est mis en scène de façon parfois forcée et de façon gênée, vague et imprécise ou au contraire de façon fière et narrative. Ainsi, si les pairs sont mentionnés lors des descriptions des lieux scolaires, c'est à des personnes extérieures au collège que les jeunes réfèrent quand ils parlent de leurs " amis " proches.

\section{Segmentation et mise en cohérence cheminée de l'expérience scolaire}

Quand il s'agit d'évoquer le parcours scolaire ou la classe, les enfants et les jeunes migrants enquêtés se représentent presque exclusivement seuls (et dans quelques cas ne se représentent pas). II importe de préciser que ces thématiques, directement liées à la vie à l'école dans ce qu'elle a de plus scolaire, sont les seules à avoir unanimement fait l'objet de représentations de soi comme seul'.

8. Quand l'auteur ne se représente pas sur les dessins ou utilise, à l'oral dans les mises en scène, les formulations impersonnelles " on " ou "ça ", nous avons, sans autre interprétation, associée cela à une forme narrative équivalente au " je ». Dans ces cas, si aucun pair n'était mentionné, nous avons apparenté cela à un récit solitaire proche de ceux dont les auteurs se représentent comme seuls. 
Illustration 3 : Dessin d'un garçon de 7 ans région nord de Paris répondant à la consigne " Dessine ta classe ".

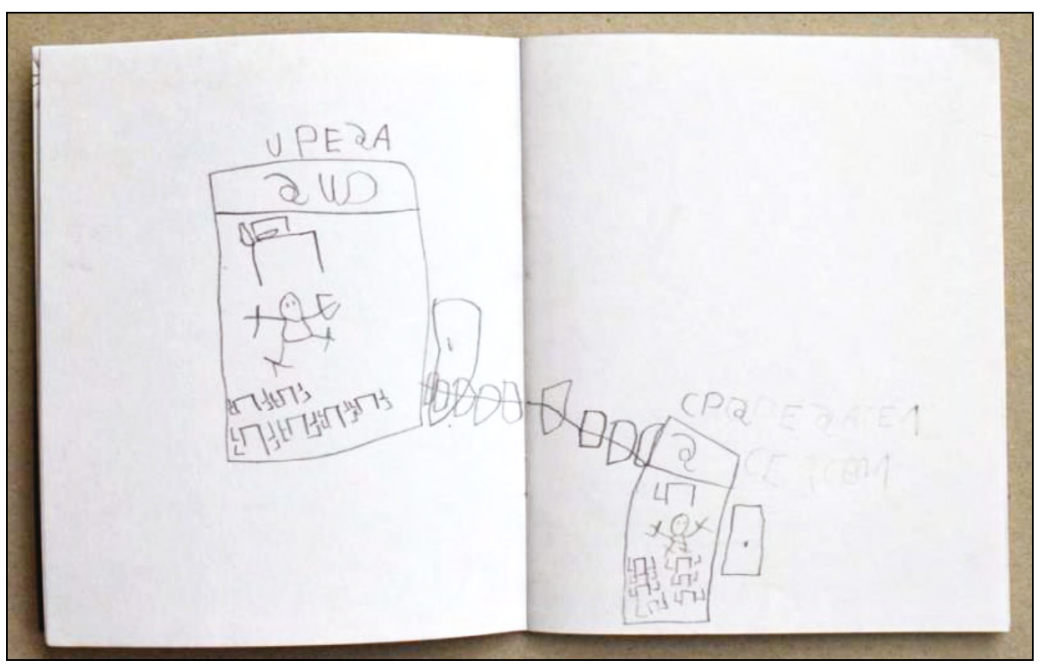

Légende de l'illustration 3: L'enfant se représente seul à deux reprises: dans sa classe et ceci en CP comme en UPE2A.

Illustration 4 : Dessin d'un garçon de 8 ans région nord de Paris répondant à la consigne "Dessine ta classe ".

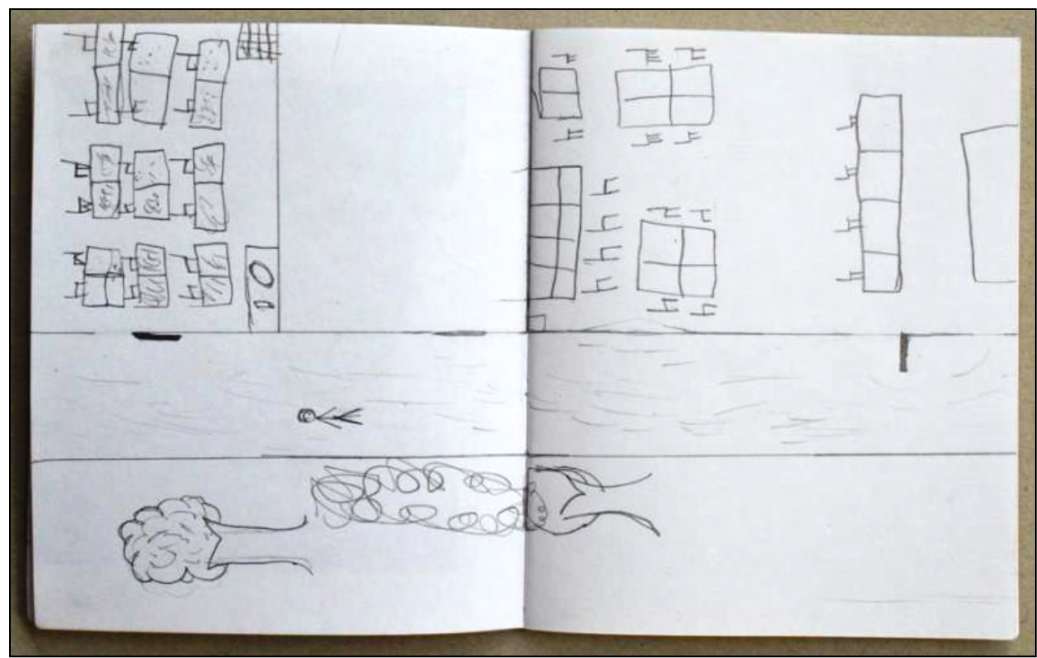

Légende de l'illustration 4: L'enfant se représente seul sur une large route allant de la classe d'UPE2A (configuration pédagogique de droite) à sa classe de rattachement (configuration pédagogique de gauche). 


\section{DOSSIER}

Cette dimension solitaire de l'expérience, dès lors qu'elle est référée au parcours scolaire, tranche avec la dimension bien plus collective des lieux scolaires, investis par les pairs. De plus, presque tous les enfants, en répondant à l'énoncé "dessine ta classe ", ont dessiné non pas une mais deux salles de classe en matérialisant sur chaque dessin un cheminement, un passage (pont, passerelle, route) entre les deux espaces de la classe de rattachement et de I'UPE2A, attestant de la transition entre deux espaces scolaires distincts et du caractère structurant de celle-ci. Ceci témoigne de l'effort de cheminement et de mise en cohérence entre différents lieux scolaires. Dans les dessins, ce cheminement n'est pas rapproché du registre de la difficulté ou de l'échec alors que de nombreux enfants sont en position scolaire délicate. Mais il est toutefois apparenté à une épreuve d'orientation qui se vit seul. Dans ces dessins, l'enseignant non plus n'est pas représenté.

\section{CONCLUSION}

Au-delà d'informer sur les enjeux proprement scolaires liés à la mise en œuvre de politiques et de pratiques scolaires liées aux enfants et aux jeunes migrants, cet article met en lumière les initiatives épistémologiques destinées à saisir l'agency des jeunes enquêtés. En concevant un cadre qui puisse se défaire d'une approche adultocentrée, le chercheur incite les jeunes enquêtés à exprimer leurs expériences socio-scolaires au moyen d'outils favorisant l'expression écrite, orale autant que corporelle. L'usage de l'entretien avec des enfants est particulière et doit parfois assumer une posture relativement directive dont nous cherchions à nous départir. En outre, les situations de migration dans lesquelles sont placés ces enfants et jeunes rendent l'usage même de l'entretien quelquefois problématique. En effet, pour nombre de ces personnes, l'entretien résonne comme une épreuve administrative, une ritualisation initiatique du parcours migratoire, assurée et séquencée par des agents des autorités publiques (Clavé-Mercier, Rigoni, 2017), ce qui est plus vrai encore dans le cas des jeunes mineurs désignés comme non-accompagnés (Uzureau, Senovilla Hernández, 2018). À ce qui constitue pour certains d'entre eux I'entretien, s'associent des enjeux immenses d'existence juridique et d'avenir. Cette place spécifique de l'entretien d'un point de vue symbolique a pour conséquence la production de discours parfois contraints et/ou liés à des anticipations par l'enquêté qui ne correspondent pas à ce que nous recherchons. C'est la raison pour laquelle nous avons développé, en parallèle d'observations suivies et de discussions avec les enfants et jeunes, ces formes d'expression. À travers ces outils et l'analyse du matériau recueilli, nous avons questionné la relation chercheur/enquêtés. Celle-ci permet de faire la lumière sur des formes méthodologiques souvent implicites ou inférées, prises dans les nécessités de l'inductivité qui n'a que rarement le temps et l'opportunité d'un regard réflexif. Ainsi se conjuguent et s'additionnent les difficultés d'une approche centrée sur l'enquêté tout en assumant l'empirisme de l'analyse ethnographique. II s'agit là de stimulants chantiers pour la recherche sociologique, mais qui restent pour une large part tributaire du doute et du tâtonnement, donc d'une posture d'humilité. 
En outre, la déconstruction-reconstruction d'une relation d'enquête pensée et balisée offre d'éclairer des mécanismes sociaux souvent minorés, passés sous silence ou mésinterprétés: la relation ambivalente et dépendante à l'enseignant, se contraindre à expliquer avoir des amis à l'école par exemple. Le rapport des élèves migrants allophones aux enseignants de classe ordinaire et à ceux d'UPE2A, tout comme la socialisation aux pairs, obéissent en effet à des mécanismes sociologiques qui ne peuvent être appréhendés qu'au moyen d'une relation d'enquête basée sur la durée et la co-construction tout autant que sur une réflexion sur ces méthodes et le matériau collecté.

Par ailleurs, le format de cette réflexion ne nous a pas permis de proposer une analyse complète des phénomènes sociaux structurants les quotidiennetés scolaires de ces publics; il nous a donc fallu faire des choix: nous n'avons par exemple pas abordé les mécanismes raciaux d'organisation sociale qui structurent certains lieux scolaires, ni les questions liées aux relations pédagogiques et d'apprentissages, autant de problématiques qui participent des configurations scolaires locales.

\section{Références}

Armagnague, M., Rigoni, I.(2016). Conduire une recherche sur la scolarisation d'élèves primo-migrants: quelques enjeux et défis socio-institutionnels. La nouvelle revue de l'adaptation et de la scolarisation, 75, 321-333.

Armagnague, M., Cossée, C., Cossée-Cruz, E., Hieronimy, S., Lallouette, N. (2017). Combiner sociologie et arts dans le recueil des données. Éléments vers une conceptualisation des méthodes artistiques dans les enquêtes qualitatives: l'exemple d'une recherche sur la scolarisation des enfants migrants. Migration Société, 167, 63-76.

Auger, N. (2010). Élèves nouvellement arrivés en France. Paris: Éditions des archives contemporaines.

Barrère, A. (2011). L'éducation buissonnière. Quand les adolescents se forment par eux-mêmes. Paris: Armand Colin.

Becker, H. S. (2001). Sociologie visuelle, photographie documentaire et photojournalisme: tout (ou presque) est affaire de contexte. Communications, 71(1), 333-351.

Becker, H. S. (1974). Photography and sociology. Studies in the Anthropology of Visual Communication, 1(1), 3-26.

Boal, A. (1996). Le Théâtre de l'opprimé. Paris: La Découverte.

Clavé-Mercier, A., Rigoni, I. (2017). Enquêter sur les migrations. Une approche qualitative. Migrations Société, 29(167), 15-28.

Cummins, J. (2009). Linguistic Interdependence and the Educational Development of Bilingual Children. Review of Educational Research, 49(2), 222-251.

Delalande, J. (2007). Des recherches sur l'enfance au profit d'une anthropologie de l'école. Éthnologie française, 4(37), 671-679.

Dubet, F. (1994). Sociologie de l'expérience. Paris: Seuil.

Dubet, F., Martuccelli, D. (1996). À l'école. Sociologie de l'expérience scolaire. Paris: Seuil. 


\section{DOSSIER}

Garcia, G., Hegel, F. (dir.). (2011). Entretiens collectifs: nouveaux usages? [Dossier]. Revue française de science politique, 61(3), 391-532.

Kaufmann, J.-C. (2016). L'entretien compréhensif. Paris: Armand Colin.

Mendonça Dias, C. (2013). Les progressions linguistiques et scolaires par les collégiens nouvellement arrivés, non ou peu scolarisés antérieurement. Recherches en didactique des langues et des cultures (RDLC): les cahiers de l'Acedle, 10(1), 159-175.

Mendonça Dias, C. (2012). Les progressions linguistiques des collégiens nouvellement arrivés en France. Villeneuve d'Ascq : ANRT.

Rigoni, I. (2017). Accueillir les élèves migrants. Dispositifs et interactions à l'école publique en France. Alterstice. Revue internationale de la recherche interculturelle (Québec), 7(1), 39-50.

Sayad, A. (2014). L'école et les enfants de l'immigration. Paris : Seuil.

Schiff, C. (dir.). (2003). Non scolarisation, déscolarisation et scolarisation partielle des migrants. Paris: Ministère de l'Éducation nationale. Programme interministériel de recherche sur les processus de déscolarisation.

Sirota, R. (2012). L'enfance au regard des sciences sociales. AnthropoChildren, 1. [En ligne]. https://popups.uliege.be:443/2034-8517/index.php?id=893.

Sirota, R. (2005). L'enfant acteur ou sujet dans la sociologie de l'enfance. Toulouse: Érès.

Tersigni, S. (2014). Grandir au prisme de l'ethnicisation en France et en Italie. Les groupes d'âge des 9-13 ans. In "Préadolescences " [dossier], Revue des sciences sociales.

Touraine, A. (1978). La voix et le regard. Paris: Seuil.

Uzureau, O., Senovilla Hernández, D. (2018). Les images de mineurs migrants comme outils d'enquête. Revue Science and Video, 7. 